\title{
Microscopy Techniques Used to Visualize Immune-Labeled Chlamydial Inclusion Membrane Proteins in 2D and 3D
}

\author{
Benjamin Crews ${ }^{1}$, Elizabeth R. Fischer ${ }^{2}$, Forrest Hoyt ${ }^{2}$, Bryan T. Hansen ${ }^{2}$ and Ted Hackstadt ${ }^{2}$ \\ 1. Whitworth University, Spokane, WA, USA. \\ 2. NIH/NIAID/Rocky Mountain Laboratories, Hamilton, MT, USA.
}

Chlamydia trachomatis is an obligate intracellular bacterium that causes a variety of human infections. Chlamydiae have a biphasic developmental cycle that takes place within a membrane bound compartment termed an inclusion. After replication in the host cell, the chlamydiae are released by lysis of the host cell or through a process by which the inclusion is extruded [1]. The extrusion process is believed to be controlled by small protein microdomains embedded in the inclusion membrane. For that reason, we sought to image these microdomains of inclusion membrane proteins (Incs) to elucidate the mechanism of chlamydial release $[2,3]$.

These microdomains are comprised of at least nine different Incs and were visualized by fluorescent microscopy associated with active Src-family kinases [4] and were visible on the cytosolic surfaces of the inclusion membranes (Fig. 1a-c). This method confirmed the presence of the microdomains, but not at the resolution of electron microscopy.

Horseradish peroxidase (HRP) secondary antibody conjugates followed by incubation with diaminobenzidine (DAB) generates electron dense material through an enzymatic reaction. [5] We used this approach to label the chlamydial protein microdomains followed by primary fixation with $2.5 \%$ glutaraldehyde, post-fixation with $1.0 \%$ osmium tetroxide/ $0.8 \%$ potassium ferricyanide prior to dehydration with ethanol embedding in Spurr's resin for transmission electron microscopy (TEM). The positive control antibody against the bacteria confirmed the reaction was successful (Fig. 2). Unfortunately given the relatively small size of the microdomains and the 3-dimensional nature of the inclusion membrane, finding infected cells with labeled microdomains in $80-100$ nanometer sections or $200 \mathrm{~nm}$ sections for electron tomography was extremely time consuming and yielded limited spatial information and virtually undetectable staining.

Focused ion beam scanning electron microscopy (FIB-SEM) allowed whole cell imaging of immunelabeled cells for better 3-dimensional localization of the microdomains HRP-DAB labeled cells were imaged in a Helios G4 UX FIB/SEM using Auto Slice and View ${ }^{\mathrm{TM}}$ Version 4.1 (Thermo Fischer Scientific, USA). FIB milling was performed at $30 \mathrm{kV}$ and $2.4 \mathrm{nA}$ with a milling thickness of $10 \mathrm{~nm}$. SEM imaging was performed at $2 \mathrm{kV}$ and $8.0 \mathrm{nA}$ with a dwell time of 3 microseconds and a scan size of $6144 \times 4096$ pixels $\left(4.07 \times 4.07 \mathrm{~nm}\right.$ ). The image stacks were aligned using Amira ${ }^{\mathrm{TM}}$ (Thermo Fischer Scientific, USA) or IMOD. The image volumes were segmented and reconstructed using Amira ${ }^{\mathrm{TM}}$ to visualize the DAB labeling (Fig. 3 a-d) [6].

References:

[1] K Hybiske and RS Stephens, PNAS 27 (2007) p. 11430.

[2] EI Lutter, C Martens and T Hackstadt, Comp Func Genom. 2012 (2012), p. 362104.

[3] P Nguyen, EI Lutter and T Hackstadt, Plos Path 3 (2018), p. e1006911. 
[4] J Mital et al., Cell Microbiol 12 (2010), p. 1235.

[5] WJ Brown and MG Farquhar, Meth. Cell Biol. 31 (1989), p. 553.

[6] This research was supported by the Intramural Research Program of the NIH, NIAID
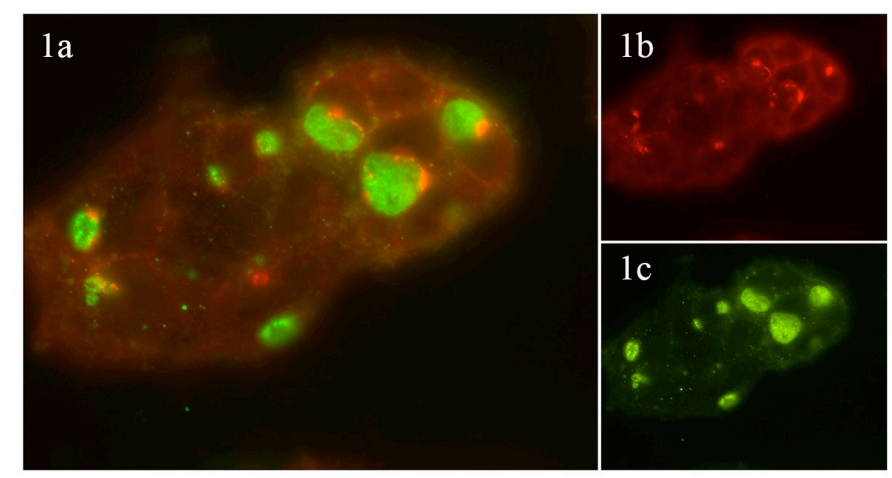

Fig. 1 Chlamydia infected HeLa cells labeled with anti-chlamydial marker (green) (c) and anti-4G10 microdomain marker (red) (b) showing association in the merged image (a) using a Nikon Eclipse 80i.
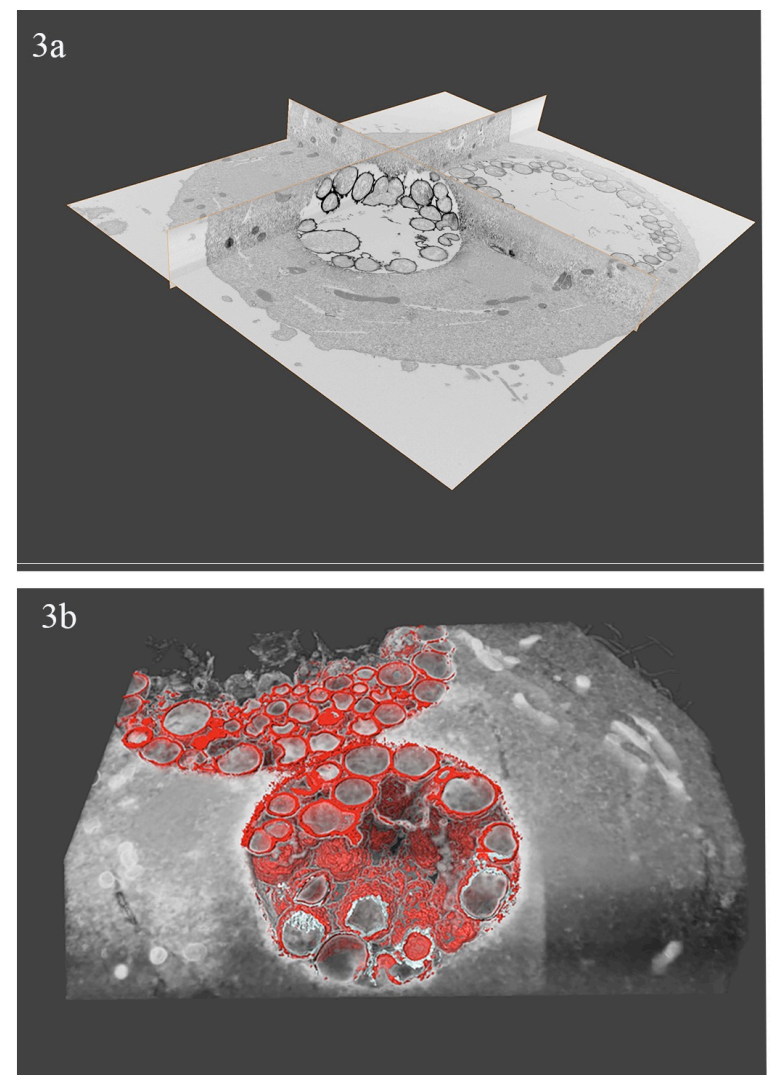

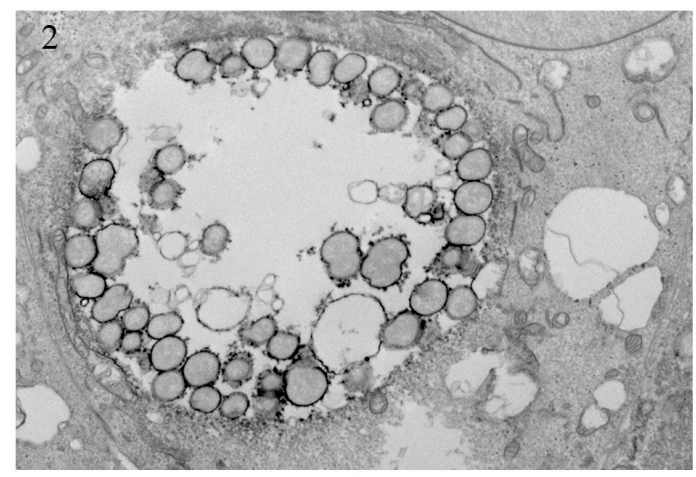

Fig. 2 Chlamydia infected HeLa cell immune labeled with anti-chlamydial surface protein followed by HRP secondary conjugate and DAB staining evident around the bacteria by TEM
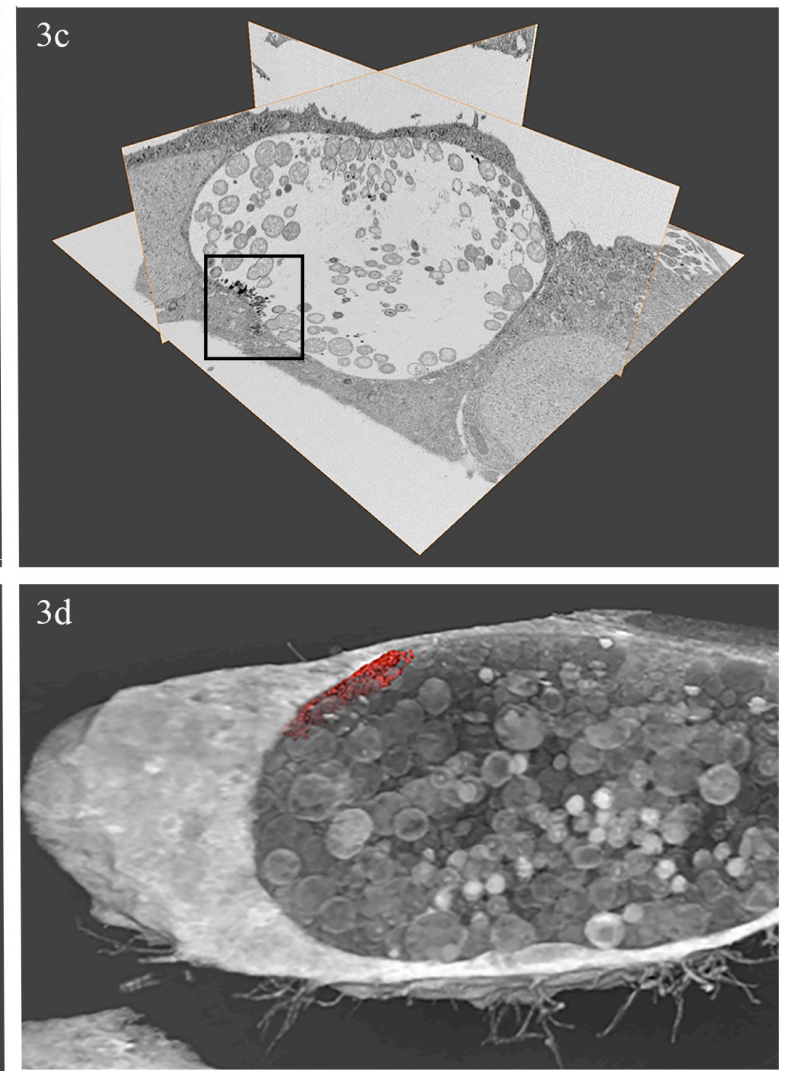

Fig. 3 Chlamydia infected HeLa cells immune labeled with anti-chlamydial surface protein (a \& b) or anti4G10 microdomain protein (c \& d) followed by HRP secondary conjugate and DAB staining evident in the reconstructions from FIB-SEM data. The orthogonal views in a \& c (box) show dense staining. The immune staining has been segmented in red for the volren views in $b \& d$. 